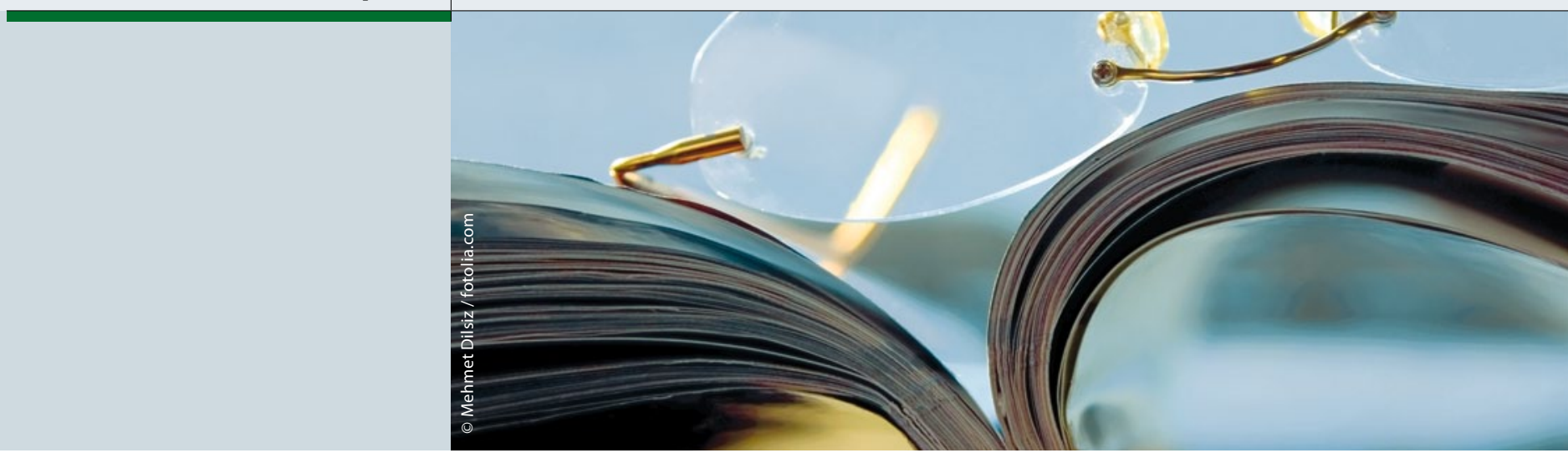

\section{PSA-Wert mit Mitte 40 prophezeit späteres Sterberisiko}

\begin{abstract}
Wie kann man unnötige PSA-Messungen und eine mögliche Überdiagnose vermeiden? US-amerikanische Urologen haben womöglich einen Weg gefunden, wie sich die Früherkennung mithilfe des Tumormarkers zielführender gestalten lässt. Gemäß ihrer Fall-Kontroll-Studie lässt sich bereits im Alter zwischen 40 und 50 Jahren am PSA-Wert ablesen, welche Männer von regelmäßigen PSA-Kontrollen profitieren würden und welche nicht.
\end{abstract}

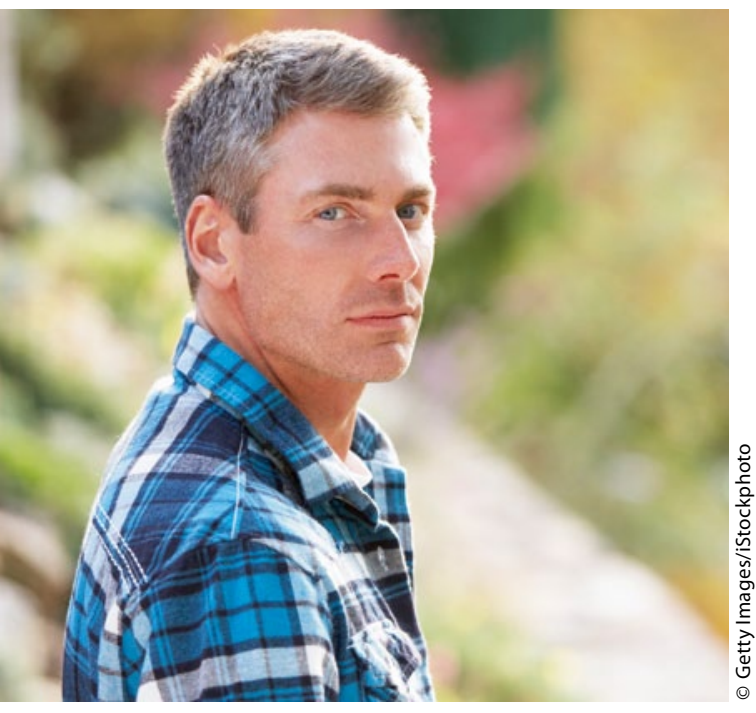

Wird er später einmal am Prostatakarzinom erkranken?

—ür eine Studie werteten Wissen- schaftler Daten von 21.277 Männern aus, die zwischen 1974 und $1984 \mathrm{im} \mathrm{Al-}$ ter von 25 bis 52 Jahren an der MalmöPreventive-Project-Studie teilgenommen hatten und deren damalige PSA (Prostata-spezifische Antigen)-Werte vorlagen. Von etwa 5.000 Männern dieser Gruppe wurde sechs Jahre später erneut das PSA gemessen. Bis Ende 2009
- also etwa 25 bis 35 Jahre später - waren laut schwedischem Krebsregister insgesamt 1.369 Männer an einem Prostatakarzinom erkrankt, davon 241 Männer an einem metastasierten Tumor, 162 waren am Krebs verstorben.

Tatsächlich stand das Risiko, im Laufe der nächsten 30 Jahre an einem lebensbedrohlichen Prostatakarzinom zu erkranken, im Zusammenhang mit der basal gemessenen PSA-Konzentration ( $\mathrm{p}$ $<0,005)$ : Fast die Hälfte (44\%; $95 \%$-CI 34-53\%) der Todesfälle entfiel auf Männer, deren Basis-PSA-Werte im Alter von 45 bis 49 Jahren im oberen Zehntel der Messwerte rangierten, bei 1,6 ng/ml oder mehr. Ähnlich hoch war der Anteil der Todesfälle (44\%; 95\%-CI 32-56\%) in der Gruppe der 51- bis 55-Jährigen mit Basis-PSA-Werten im oberen Zehntel, ab 2,4 ng/ml.

Zwar war das Langzeitrisiko für ein metastasiertes Prostatakarzinom bei PSA-Werten unterhalb des Medians der jeweiligen Altersgruppe (45-49 Jahre: 0,68 ng/ml; 51-55 Jahre: 0,85 ng/ml) nicht gleich null, doch blieb es gering. Bei den 45- bis 49-Jährigen betrug das 15-Jahres-Risiko 0,09\% (95\%-CI 0,03$0,23 \%$ ), bei den 51 - bis 55-Jährigen $0,28 \%$ (95\%-CI 0,11-0,66\%).
Diese Daten seien umso bedeutender, da sie in einer Zeit generiert worden sind, in der regelmäßige PSA-Untersuchungen eher die Ausnahme als die Regel waren, betonen die Autoren. Nach ihrer Ansicht könnten diese Ergebnisse unter Berücksichtigung des bereits früher bestätigten minimalen Langzeitrisikos bei Männern um die 60 mit PSA-Werten unter $1 \mathrm{ng} / \mathrm{ml}$ dazu beitragen, neue Screeningmodelle zu entwickeln. Die Autoren empfehlen, den PSA-Wert Mitte bis Ende 40 erstmals zu messen. Bei Werten unter $1,0 \mu \mathrm{g} / 1$ sollten die Männer mit Anfang 50 und mit Anfang 60 wiederkommen. Bei höheren Werten sind Kontrollen im zweijährigen Turnus erforderlich. Männer, die auch mit 60 PSA-Konzentrationen unterhalb des Medians aufweisen, müssten nicht mehr zur Vorsorge einbestellt werden. Sind die Werte mit 60 jedoch höher, ist das Screening bis zum 70. Lebensjahr fortzuführen. Bei beinahe der Hälfte aller Männer, davon sind die Studienautoren überzeugt, genügen demnach drei PSA-Tests: einer mit Mitte 40, einer Anfang 50 und einer Anfang 60.

Fazit: Anhand des PSA-Wertes im vierten oder fünften Lebensjahrzehnt können diejenigen Männer identifiziert werden, die sehr wahrscheinlich im späteren Leben an einem lebensbedrohlichen Prostatakarzinom erkranken werden und somit von regelmäßigen Kontrolluntersuchungen tatsächlich profitieren würden.

Dr. Dagmar Kraus

Vickers AJ et al. Strategy for detection of prostate cancer based on relation between prostate specific antigen at age 40-55 and long term risk of metastasis: case-control study. BMJ 2013; 346: $f 2023$ 